\title{
Numerical homogenization of nonlinear viscoplastic two-dimensional polycrystals
}

\author{
FRÉDÉRIC LEGOLL \\ CERMICS, Ecole Nationale des Ponts et Chaussées, 6 et 8 av. B. Pascal, Cité Descartes \\ 77455 Marne la Vallée, Cedex 2, France and EDF R\&D \\ Analyse et Modèles Numériques, 1 av. du Général de Gaulle, 92140 Clamart, France \\ E-mail: legoll@cermics.enpc.fr
}

\begin{abstract}
In this article, we numerically determine the effective stress-strain relation of some two-dimensional polycrystals. These are aggregates of a few tens of perfectly bonded singlecrystal (hexagonal atomic lattice) grains, with varying orientations. Each grain obeys a given nonlinear viscoplastic stress-strain relation, which depends on the orientation of the grain. Precise calculations performed with this microscopic model are compared with calculations done with a macroscopic approximate model (in which matter has no microstructure) in order to determine the macroscopic constitutive law. We find an effective behaviour for the stationary response which appears to be also consistent for the transient response. The influence of the number of grains as well as that of the distribution of grain orientations are investigated.
\end{abstract}

Mathematical subject classification: 74Q15,74D10.

Key words: numerical homogenization, polycrystal, effective constitutive law, finite element method.

\section{Introduction}

The theoretical prediction of the effective response of a heterogeneous material is still an essentially open question. In some few simple cases, an analytic closed form expression is known. For instance, this is the case for a linear elastic matrix with linear elastic inclusions, in the dilute limit (that is, inclusions are considered too far away from one another to have an interaction) [7].

\#568/03. Received: 15/IV/03. Accepted: 03/X/03. 
A more general case is that of a material presenting material nonlinearity $[6,10]$. The constitutive law (also named the stress-strain relation) of such a material reads

$$
\varepsilon(x, t)=\frac{\partial U}{\partial \sigma}(x, \sigma(x, t)),
$$

where the real-valued function $U$ is the heterogeneous elastic stress potential, $\sigma(x, t)$ is the stress tensor, and $\varepsilon(x, t)$ is the strain tensor. Throughout this article, we work under the assumption of small perturbations (small strain, small displacement). All fields are defined on the reference configuration, and $\varepsilon(x, t)$ is linked to the displacement field $u(x, t)$ by the linearized compatibility equation

$$
\varepsilon=\frac{1}{2}\left(\nabla u+\nabla u^{T}\right) .
$$

In this setting, one can derive various bounds and estimates on the effective behaviour $[4,8,11,12,13]$. Let us note that, in general, no closed form expression for the effective elastic stress potential is available.

In this article, we will consider the elasto-viscoplastic materials whose constitutive law reads

$$
\dot{\varepsilon}(x, t)=\frac{\partial U^{v p}}{\partial \sigma}(x, \sigma(x, t))+\frac{\partial U^{e}}{\partial \dot{\sigma}}(x, \dot{\sigma}(x, t)),
$$

where $U^{v p}$ is the viscoplastic stress potential (also referred to as the dissipation potential) and $U^{e}$ is the elastic stress potential. So the strain rate tensor $\dot{\varepsilon}(x, t)$, which is the time derivative of the strain tensor, depends both on the stress tensor $\sigma$ and the stress rate tensor $\dot{\sigma}$. In such a case, when the stress-strain relation cannot be written with a unique potential, there are no theoretical bounds known.

In the following, we numerically investigate the effective behaviour of a heterogeneous polycrystal obeying such an elasto-viscoplastic law [1]. With a view to studying a more realistic and complex model in the future, we want to check here whether an effective constitutive law of type (3) can be inferred from the examination of the material at lower scale.

The article is organized as follows. The polycrystal model is presented in Section 2. Let us just mention in this Introduction that a polycrystal is an aggregate of perfectly bonded single-crystal grains, and that each grain is homogeneous 
and obeys a given nonlinear stress-strain relation. This relation depends on parameters which are not the same from one grain to another one, thus making the polycrystal heterogeneous. Section 3 is dedicated to the theoretical study of such a heterogeneous law. We first recall some definitions and classical results on the derivation of an effective law for heterogeneous materials, by a homogenization procedure. As above stated, the classical procedure does not apply for our model, since the microscopic law cannot be written by using a single potential. We however decide to make use of the classical procedure separately on the elastic potential and on the viscoplastic potential, thus obtaining an effective elastic potential and an effective viscoplastic potential, up to some unknown parameters. Collecting these two effective potentials, we are able to postulate some expression for the effective constitutive law (see (17) below).

Our aim is to use, in the future, the effective law in the following way. Computing the response of a structure (composed of a large number of grains) by using the microscopic law is very expensive. Recall that, if one uses a finite element method, the mesh size has to be smaller than the grain size. Using an effective homogeneous law is much cheaper, for it allows for larger mesh sizes. In this article, as a first step, we look for an effective constitutive law which is consistent with the microscopic law. This consistency is checked by comparing the numerical results that are obtained on the basis of the effective law with the numerical results that are obtained (through a costly calculation) with the microscopic law. For this purpose, we choose some test problems, and make two computations, one with the macroscopic model, one with the microscopic model (by using a very fine finite element mesh). Numerical results are given in Section 4 .

\section{The microscopic model}

The materials we deal with are metals that have a hexagonal atomic lattice (see Fig. 1). The orientation of the lattice is not uniform in the material: by definition, a grain is a domain of the material in which the orientation stays constant, and a polycrystal is a set of a large number of perfectly-bonded grains [1]. We will only consider polycrystals made of grains of isotropic shape (there is no special direction in the grain shape). For the materials we deal with, the characteristic 
size of a grain is $5 \cdot 10^{-6} \mathrm{~m}$, which is much larger than the atomic scale $\left(10^{-10} \mathrm{~m}\right)$ : so it is possible to use a continuum model to describe the constitutive relation inside a grain. At this scale, the stress tensor is $\sigma_{\mu}(x, t)$, the displacement is $u_{\mu}(x, t)$ and the strain tensor $\varepsilon_{\mu}(x, t)$ is linked to the displacement by the linearized compatibility equation (2). We do not include in our model any grain interface properties, and we only suppose that the displacement and the stress vector are continuous at the grain interfaces. The stress-strain relation inside a grain depends on its orientation, and the heterogeneity in the polycrystal comes from the fact that this orientation is not the same from one grain to another one.
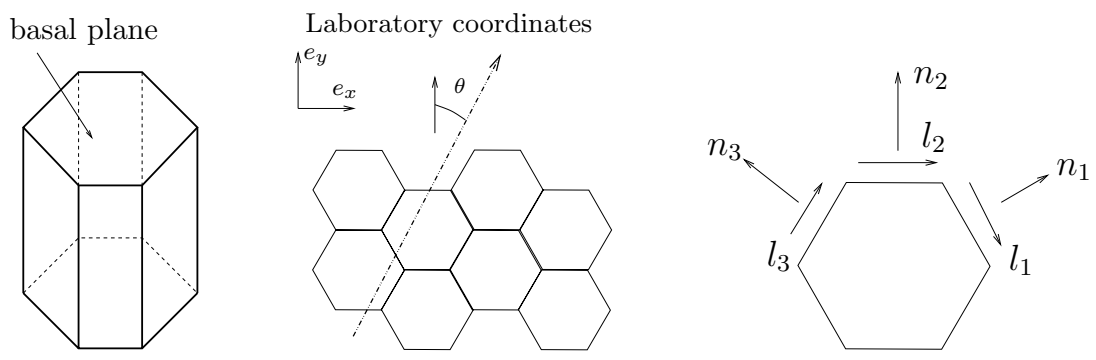

Figure 1 - Atomic lattice inside a grain: 3D unit cell (left), 2D section along the basal plane inside a grain (center), the 3 slip systems we take into account (right). The orientation of the grain is given by the angle $\theta$.

We suppose in the following that for all grains, the basal plane of the atomic lattice (see Fig. 1) is the same, namely the $\left(e_{x}, e_{y}\right)$ plane. So, the grain orientation is defined by an angle between 0 and $\pi / 6$. We also assume that the grain orientations occur with equal probability (there are actually very few experimental data for the metals we deal with, so this assumption is the most sensible one).

Let us now write the stress-strain relation inside a grain. In the metals we study, there are 12 preferred slip systems, defined by the plane in which the slip takes place (the normal direction to this plane is denoted by $n_{s}$ ), and by the slip direction $l_{s}$. Here, the vectors $n_{s}(x)$ and $l_{s}(x)$ depend on the space variable $x$, as they change from one grain to another one. In this article, we want to work in a 2D geometry in the $\left(e_{x}, e_{y}\right)$ plane, so we only take into account the 3 systems for which the vectors $n_{s}(x)$ and $l_{s}(x)$ belong to the $\left(e_{x}, e_{y}\right)$ plane (see Fig. 1). Knowing the slip systems, one can compute the orientation tensors $m_{s}(x)$, which 
are defined by

$$
m_{s}(x)=\frac{1}{2}\left(n_{s}(x) \otimes l_{s}(x)+l_{s}(x) \otimes n_{s}(x)\right) .
$$

The strain rate tensor $\dot{\varepsilon}_{\mu}$ is the sum of two terms, the elastic strain rate tensor $\dot{\varepsilon}_{\mu}^{e}$ and the viscoplastic strain rate tensor $\dot{\varepsilon}_{\mu}^{v p}$. The elastic term is given by the linear Hooke law. We do not include in our model any nonlinear elastic effects, for they are small in comparison to the efforts we account for. We suppose that the elastic characteristics are homogeneous and isotropic in the polycrystal. Using the Young modulus $E$ and the Poisson ratio $v$, the elastic term reads

$$
\varepsilon_{\mu}^{e}(x, t)=\frac{1+v}{E} \sigma_{\mu}(x, t)-\left(\frac{v}{E} \operatorname{tr} \sigma_{\mu}(x, t)\right) I,
$$

where $I$ is the identity $3 \times 3$ tensor. On the other hand, we assume the viscoplastic term to be of a power-law type

$$
\dot{\varepsilon}_{\mu}^{v p}(x, t)=\sum_{s=1}^{3}\left(\frac{\left|\sigma_{\mu}(x, t): m_{s}(x)\right|}{K_{\mu}}\right)^{n} \operatorname{sign}\left(\sigma_{\mu}(x, t): m_{s}(x)\right) m_{s}(x) .
$$

We make the assumption that the parameters $n$ and $K_{\mu}$ of the power-law are the same for all grains. So, as mentioned above, the heterogeneity from one grain to another one just comes from the fact that the orientation tensors $m_{s}(x)$ are not the same.

So, the constitutive relation inside a grain reads

$$
\dot{\varepsilon}_{\mu}(x, t)=\dot{\varepsilon}_{\mu}^{e}(x, t)+\dot{\varepsilon}_{\mu}^{v p}(x, t) .
$$

Recasting (7) in the form of (3), we see that, in our case, the microscopic stress potentials (introduced in (3)) read

$$
\begin{aligned}
U_{\mu}^{e}\left(\dot{\sigma}_{\mu}\right) & =\frac{1}{2} \dot{\sigma}_{\mu} \cdot \Lambda \cdot \dot{\sigma}_{\mu}, \\
U_{\mu}^{v p}\left(x, \sigma_{\mu}\right) & =\frac{1}{n+1}\left(\frac{1}{K_{\mu}}\right)^{n} \sum_{s=1}^{3}\left|\sigma_{\mu}: m_{s}(x)\right|^{n+1},
\end{aligned}
$$

where the fourth order tensor $\Lambda$ only depends on $E$ and $v$ (see (5)). 
Let $\Omega$ be the region occupied by the polycrystal in the reference configuration. Solving the microscopic model consists in searching for the displacement field $u_{\mu}(x, t)$ solution to the equilibrium equation

$$
\forall x \in \Omega, \forall t \in[0, T], \quad \operatorname{div} \sigma_{\mu}(x, t)=0,
$$

along with constitutive laws (5-6-7), compatibility equation (2) and convenient initial and boundary conditions.

Quantitatively, we use the following numerical values:

$$
E=105000 \mathrm{MPa}, \quad v=0.43, \quad K_{\mu}=178 \mathrm{MPa}, \quad n=6.5 .
$$

\section{The homogenization procedure}

In Section 3.1, we first briefly recall the classical homogenization procedure [12] used in the stationary case when the stress-strain relation can be written by using a single potential. Next, in Section 3.2, we use the procedure to determine the analytical expression, up to some parameters, of the effective behaviour of the polycrystal. Henceforth, there are no body forces.

\subsection{Classical homogenization procedure}

Let us consider an elastic material (see Section 1) in the stationary case, described by a heterogeneous microscopic stress potential $U_{\mu}\left(x, \sigma_{\mu}\right)$. The constitutive law is given by (1) with $U \equiv U_{\mu}$. We suppose that $U_{\mu}$ is strictly convex with respect to $\sigma_{\mu}$. The microscopic deformation potential $W_{\mu}\left(x, \varepsilon_{\mu}\right)$ is defined as the Legendre transform of $U_{\mu}$ with respect to $\sigma_{\mu}$.

We can first work with the displacement as the unknown and define the socalled effective deformation potential $W_{M}$. For a given symmetric constant tensor $\varepsilon_{M}, W_{M}\left(\varepsilon_{M}\right)$ is defined by

$$
W_{M}\left(\varepsilon_{M}\right)=\inf \left\{\left\langle W_{\mu}\left(x, \varepsilon_{\mu}(x)\right)\right\rangle, \varepsilon_{\mu}(x) \in K\left(\varepsilon_{M}\right)\right\},
$$

where $\langle\cdot\rangle$ is the average over $\Omega$ and the minimization space is defined by

$$
K\left(\varepsilon_{M}\right)=\left\{\varepsilon_{\mu}(x) ; \exists u_{\mu}(x) \text { satisfying }(2) \text { in } \Omega, u_{\mu}(x)=\varepsilon_{M} \cdot x \text { on } \partial \Omega\right\} .
$$


Note that, as a consequence of (2), all strain tensors $\varepsilon_{\mu}$ in $K\left(\varepsilon_{M}\right)$ satisfy $\left\langle\varepsilon_{\mu}(x)\right\rangle=\varepsilon_{M}$. Let $\bar{\varepsilon}_{\mu}(x)$ be the minimizer of problem (11), and let $\bar{\sigma}_{\mu}(x)$ be the microscopic stress field at equilibrium. The effective strain and stress tensors are defined as the averages over $\Omega$ of the microscopic tensors. We have already noticed that the effective strain tensor is $\varepsilon_{M}$. We set $\sigma_{M}=\left\langle\bar{\sigma}_{\mu}(x)\right\rangle$. One can show that effective tensors and potential are linked by

$$
\sigma_{M}=\frac{\partial W_{M}}{\partial \varepsilon_{M}}\left(\varepsilon_{M}\right)
$$

For completeness, let us mention that there are other ways to define an effective potential. We have so far worked with the deformation potential $W_{\mu}\left(x, \varepsilon_{\mu}\right)$, we may alternatively work with the stress potential $U_{\mu}\left(x, \sigma_{\mu}\right)$, the stress field being the unknown. The so-called effective stress potential $U_{M}$ is defined by

$$
U_{M}\left(\sigma_{M}\right)=\inf \left\{\left\langle U_{\mu}\left(x, \sigma_{\mu}(x)\right)\right\rangle, \sigma_{\mu}(x) \in S\left(\sigma_{M}\right)\right\},
$$

where $\sigma_{M}$ is a given symmetric constant tensor, and where the minimization space is defined by

$$
S\left(\sigma_{M}\right)=\left\{\sigma_{\mu}(x) ; \sigma_{\mu}(x) \cdot n(x)=\sigma_{M} \cdot n(x) \text { on } \partial \Omega, \operatorname{div} \sigma_{\mu}=0 \text { in } \Omega\right\} .
$$

Let $\bar{\sigma}_{\mu}(x)$ be the minimizer of problem (13), and let $\bar{\varepsilon}_{\mu}(x)$ be the microscopic strain field at equilibrium, which is related to $\bar{\sigma}_{\mu}(x)$ by (1) where $U$ is replaced by $U_{\mu}$. Again, effective tensors are defined as averages over $\Omega$ of microscopic tensors. All stress tensors $\sigma_{\mu}(x)$ in $S\left(\sigma_{M}\right)$ satisfy $\left\langle\sigma_{\mu}(x)\right\rangle=\sigma_{M}$, so the effective stress tensor is $\sigma_{M}$. We set $\varepsilon_{M}=\left\langle\bar{\varepsilon}_{\mu}(x)\right\rangle$. As in the first case, one can show that effective tensors and potential are linked by $\varepsilon_{M}=\frac{\partial U_{M}}{\partial \sigma_{M}}\left(\sigma_{M}\right)$.

One says that the material follows an effective stress-strain relation if the effective stress potential $U_{M}$ defined by (13) is the Legendre transform, with respect to the macroscopic strain tensor $\varepsilon_{M}$, of the effective deformation potential $W_{M}$ defined by (11).

Remark. The homogenization procedure we have just recalled is based on calculus of variations, and no quantity depends on time. In the time-dependent case, under the quasistatic approximation, it is also possible to define an effective deformation potential and an effective stress potential, by the same procedure as above. 


\subsection{Homogenization of the polycrystal law}

We now proceed to the homogenization of the polycrystal model presented in Section 2. Constitutive laws are (5-6-7), corresponding potentials are defined by (8-9), and the equilibrium equation is (10). When writing this equation, we have neglected the acceleration. As two potentials are involved, and as the constitutive law is time-dependent, we cannot directly use the theory we have just recalled. However, we can apply the theory separately on the elastic stress potential and on the viscoplastic stress potential. Indeed, if we only consider one potential, we are in the setting detailed in Section 3.1. Actually, the procedure is immediate for the elastic potential as elastic properties are homogeneous in the polycrystal. We thus focus on the viscoplastic stress potential. To simplify notation, let $d_{\mu}=\dot{\varepsilon}_{\mu}^{v p}$ denote the microscopic viscoplastic strain rate tensor.

\subsubsection{The viscoplastic term}

We first note that tensors $m_{s}$ are symmetric and satisfy $m_{s}^{x x}=-m_{s}^{y y}$ and $m_{s}^{x z}=$ $m_{s}^{y z}=m_{s}^{z z}=0$ (see (4)). Hence, for any symmetric microscopic stress tensor $\sigma_{\mu}$, we have $\sigma_{\mu}(x): m_{s}(x)=\alpha_{\mu}(x) u_{s}(x)+\beta_{\mu}(x) v_{s}(x)$, where we set

$$
\alpha_{\mu}=\sigma_{\mu}^{x x}-\sigma_{\mu}^{y y}, \quad \beta_{\mu}=2 \sigma_{\mu}^{x y}, \quad u_{s}=m_{s}^{x x}, \quad v_{s}=m_{s}^{x y} .
$$

With (6), we note that tensors $d_{\mu}$ only depend on two scalar variables, $d_{\mu}^{x x}$ and $d_{\mu}^{x y}$. So the only variables to consider are $d_{\mu}^{x x}, d_{\mu}^{x y}, \alpha_{\mu}$ and $\beta_{\mu}$. The potential $U_{\mu}^{v p}\left(x, \sigma_{\mu}\right)$ that we introduced in (9) is not strictly convex with respect to $\sigma_{\mu}$, but if we rewrite it in terms of $\left(\alpha_{\mu}, \beta_{\mu}\right)$,

$$
U_{\mu}^{v p}\left(x, \alpha_{\mu}, \beta_{\mu}\right)=\frac{1}{n+1}\left(\frac{1}{K_{\mu}}\right)^{n} \sum_{s=1}^{3}\left|\alpha_{\mu} u_{s}(x)+\beta_{\mu} v_{s}(x)\right|^{n+1},
$$

it turns out to be a strictly convex function of $\left(\alpha_{\mu}, \beta_{\mu}\right)$, and (6) can be recast into

$$
d_{\mu}^{x x}=\frac{\partial U_{\mu}^{v p}}{\partial \alpha_{\mu}} \quad \text { and } \quad d_{\mu}^{x y}=\frac{\partial U_{\mu}^{v p}}{\partial \beta_{\mu}} .
$$

Let $W_{\mu}^{v p}\left(x, d_{\mu}^{x x}, d_{\mu}^{x y}\right)$ be the Legendre transform of $U_{\mu}^{v p}$ with respect to $\left(\alpha_{\mu}, \beta_{\mu}\right)$. As $U_{\mu}^{v p}$ is a homogeneous function of degree $n+1$ of the pair $\left(\alpha_{\mu}, \beta_{\mu}\right)$, the potential $W_{\mu}^{v p}$ is a homogeneous function of degree $1+1 / n$ of the pair $\left(d_{\mu}^{x x}, d_{\mu}^{x y}\right)$. 
We now turn to the derivation of an effective model. Following the general procedure recalled in Section 3.1, we define the effective potential $W_{M}^{v p}$ by

$$
W_{M}^{v p}\left(d_{M}^{x x}, d_{M}^{x y}\right)=\inf \left\{\left\langle W_{\mu}^{v p}\left(x, d_{\mu}^{x x}, d_{\mu}^{x y}\right)\right\rangle,\left(d_{\mu}^{x x}, d_{\mu}^{x y}\right) \in K\left(d_{M}^{x x}, d_{M}^{x y}\right)\right\},
$$

where $K\left(d_{M}^{x x}, d_{M}^{x y}\right)$ is defined by

$K\left(d_{M}^{x x}, d_{M}^{x y}\right)=\left\{\begin{array}{c}\left(d_{\mu}^{x x}(x), d_{\mu}^{x y}(x)\right) ; \exists u_{\mu}(x) \text { such that } u_{\mu}(x)=\gamma\left(d_{M}^{x x}, d_{M}^{x y}\right) \cdot x \\ \text { on } \partial \Omega \text { and } \frac{1}{2}\left(\nabla u_{\mu}+\nabla u_{\mu}^{T}\right)=\gamma\left(d_{\mu}^{x x}(x), d_{\mu}^{x y}(x)\right) \text { in } \Omega\end{array}\right\}$,

the function $\gamma$ being defined by

$$
\gamma:(u, v) \in \mathbb{R}^{2} \mapsto\left(\begin{array}{ccc}
u & v & 0 \\
v & -u & 0 \\
0 & 0 & 0
\end{array}\right) \in \mathcal{M}_{3}(\mathbb{R})
$$

Just as (12) holds, it holds that

$$
\alpha_{M}=\sigma_{M}^{x x}-\sigma_{M}^{y y}=\frac{\partial W_{M}^{v p}}{\partial d_{M}^{x x}}, \quad \beta_{M}=2 \sigma_{M}^{x y}=\frac{\partial W_{M}^{v p}}{\partial d_{M}^{x y}} .
$$

The macroscopic potential $W_{M}^{v p}$ is a homogeneous function of degree $1+1 / n$ of $\left(d_{M}^{x x}, d_{M}^{x y}\right)$. To use this fact, we need to change variables: instead of working with the cartesian variables $d_{M}^{x x}$ and $d_{M}^{x y}$, let us work with the polar coordinates associated to them, the radius

$$
R_{M}=\sqrt{\left(d_{M}^{x x}\right)^{2}+\left(d_{M}^{x y}\right)^{2}}
$$

and the angle $\theta_{M}$. These variables present the advantage that $R_{M}$ and $\theta_{M}$ are respectively homogeneous functions of degree 1 and 0 of $\left(d_{M}^{x x}, d_{M}^{x y}\right)$. So $W_{M}^{v p}$ reads

$$
W_{M}^{v p}\left(R_{M}, \theta_{M}\right)=R_{M}^{1+1 / n} C\left(\theta_{M}\right)
$$

where $C$ is an unknown function.

As this point, we introduce the following simplification. Considering that, first, all grain orientations occur with equal probability, and second, that the geometry of the grains and of the polycrystal is isotropic, we postulate, without any rigorous 
justification of this fact, that the response of the polycrystal is isotropic, at least when the number of grains is large enough. We therefore simplify the previous expression of $W_{M}^{v p}$, setting $C\left(\theta_{M}\right)$ as an (unknown) constant $C$, for $\theta_{M}$ is an anisotropic variable whereas $R_{M}$ is an isotropic variable.

Let us define

$$
J\left(\sigma_{M}\right)=\sqrt{\frac{3}{2}} \sqrt{\left(\tilde{\sigma}_{M}^{x x}\right)^{2}+\left(\tilde{\sigma}_{M}^{y y}\right)^{2}-\frac{1}{2}\left(\tilde{\sigma}_{M}^{z z}\right)^{2}+2\left(\sigma_{M}^{x y}\right)^{2}},
$$

where $\tilde{\sigma}_{M}=\sigma_{M}-\left(\frac{1}{3} \operatorname{tr} \sigma_{M}\right) 1$ is the deviatoric part of $\sigma_{M}$. Then equations (14) can be written as

$$
\dot{\varepsilon}_{M}^{v p}=\left(\frac{J\left(\sigma_{M}\right)}{K_{M}}\right)^{n} \frac{\partial J}{\partial \sigma_{M}}
$$

where $K_{M}$ is an unknown parameter (playing the role of the constant $C$ used above) that we will determine by numerical computations in Section 4.

\subsubsection{Postulated macroscopic model for the polycrystal}

In the previous part, we have made use of the classical homogenization procedure to obtain separately an elastic effective potential and a viscoplastic effective potential. We postulate, again without any rigorous justification of this fact, that the effective constitutive law for the polycrystal is the sum of the elastic effective term with the viscoplastic effective term. So the effective constitutive law that we use is

$$
\dot{\varepsilon}_{M}(x, t)=\Lambda: \dot{\sigma}_{M}(x, t)+\left(\frac{J\left(\sigma_{M}(x, t)\right)}{K_{M}}\right)^{n} \frac{\partial J}{\partial \sigma_{M}},
$$

where $J$ is defined by (15). Solving the effective model consists in searching for the displacement field $u_{M}(x, t)$ solution to the equilibrium equation

$$
\forall x \in \Omega, \forall t \in[0, T], \quad \operatorname{div} \sigma_{M}(x, t)=0,
$$

along with constitutive law (17), compatibility equation (2) and convenient initial and boundary conditions.

The whole microscopic constitutive law involves an ODE, and the procedures detailed in Section 3.1 do not apply in this case. With the numerical tests described in the following, we check whether this approximation may be sensible. 


\section{Numerical results}

In the previous section, working with the deformation potential, we have found an effective model for the polycrystal, up to the knowledge of the constant $K_{M}$ (see (17)). In order to determine a value for $K_{M}$, we use numerical computations on different polycrystals [2,5], with several linear displacement boundary conditions. In the following, we check that there exists a single value for $K_{M}$ such that macroscopic computations agree with microscopic computations for all test problems (that is, macroscopic tensors are equal to the average of microscopic tensors over the polycrystal $\Omega$ ).

One can also work with the microscopic stress potential to obtain an effective stress potential. One finds the same result as (16), with a a priori different constant $K_{M}^{s}$. To numerically determine a value for $K_{M}^{s}$, one would follow the same procedure as before, except that one would work with linear surface force boundary conditions. If the value found for $K_{M}^{s}$ is the same as the value found for $K_{M}$ (with linear displacement boundary conditions), then the effective stress potential is the Legendre transform of the effective deformation potential, and the polycrystal actually obeys an effective stress-strain relation (see Section 3.1). We did not make this kind of test, since, when one uses surface force boundary conditions, the displacement at equilibrium is only determined up to a rigid body motion.

Finally, a third test is possible: one can use mixed boundary conditions (we impose displacement on some part on the boundary and surface force elsewhere). Results of this kind of test are given in the following. The polycrystal actually obeys an effective stress-strain relation if the value previously found for $K_{M}$ (using linear displacement boundary conditions) is also valid with these mixed boundary conditions.

We have performed numerical tests with three different polycrystals, one of 30 grains (first with a coarse mesh: 5 to 15 finite elements per grain; then with a finer mesh: finite element edges two times smaller), and two of 110 grains (the same grain geometry, but with two different orientation samples). We work in generalized plane strain in direction $z$, that is to say we just simulate a 2D layer of the polycrystal of side surface $S$, with 3D displacement, strain and stress tensor fields. Shears $\varepsilon_{x z}$ and $\varepsilon_{y z}$ are equal to zero, and $\varepsilon_{z z}$ is uniform on the whole layer. 
The value of $\varepsilon_{z z}$ is obtained by assuming that the resulting force normal to the layer is zero.

On the side surface $S$, we choose several different boundary conditions: linear displacement boundary conditions (tension compression, thus a strain denoted by a superscript TC; shear, a strain denoted S; tension compression shear, a strain denoted TCS), and also mixed boundary conditions, letting two opposite faces force free, imposing zero normal displacement on one face, and imposing a uniform tensile displacement rate on the last face (test denoted T). For displacement boundary conditions, the strain tensors are

$$
\varepsilon_{M}^{T C}(t)=\left(\begin{array}{ccc}
\alpha t & 0 & 0 \\
0 & -\alpha t & 0 \\
0 & 0 & 0
\end{array}\right), \quad \varepsilon_{M}^{S}(t)=\left(\begin{array}{ccc}
0 & \alpha t & 0 \\
\alpha t & 0 & 0 \\
0 & 0 & 0
\end{array}\right)
$$

and

$$
\varepsilon_{M}^{T C S}(t)=\left(\begin{array}{ccc}
\alpha_{1} t & \alpha_{2} t & 0 \\
\alpha_{2} t & -\alpha_{1} t & 0 \\
0 & 0 & 0
\end{array}\right)
$$

For brevity, we only detail here one test case, namely that of a polycrystal subjected to shear load. The averaged microscopic strain tensor and the macroscopic strain tensor increase linearly as time increases. One can see on Fig. 2 the averaged microscopic stress $\left\langle\sigma_{\mu}(x, t)\right\rangle$ as a function of time (we have $\left\langle\sigma_{\mu}^{y y}\right\rangle=-\left\langle\sigma_{\mu}^{x x}\right\rangle$ and $\left.\sigma_{\mu}^{z x}(x, t)=\sigma_{\mu}^{z y}(x, t)=\sigma_{\mu}^{z z}(x, t)=0\right)$, and the macroscopic stress $\sigma_{M}(t)$, which is uniform in this case. We make the assumption that, in the long-time limit, the stress tensors $\sigma_{\mu}(x, t)$ and $\sigma_{M}(x, t)$ converge to a limit, which thus corresponds to the stationary regime of (7) and (17). One can check that the $\operatorname{limit}_{t \rightarrow \infty} \sigma_{M}^{x y}(t)$ depends on $K_{M}$ (for this shear load test, an analytical expression can be found). We choose $K_{M}$ so that

$$
\lim _{t \rightarrow \infty} \sigma_{M}^{x y}(t)=\lim _{t \rightarrow \infty}\left\langle\sigma_{\mu}^{x y}(x, t)\right\rangle,
$$

which leads in this case to the numerical value $K_{M}=347 \mathrm{MPa}$. The previous equation enforces that, in the long-time limit, the effective law is consistent with the microscopic law. The macroscopic stress displayed on Fig. 2 has been 

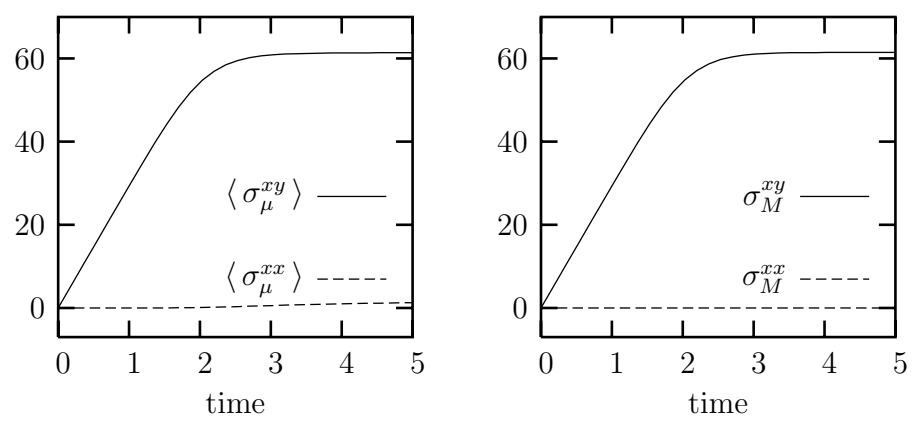

Figure 2 - Shear load on the 30 grain polycrystal: averaged microscopic stress (left), macroscopic stress (right).

computed using this value of $K_{M}$. We also notice that $\sigma_{M}^{x x}=0$, as expected. On the other hand, $\left\langle\sigma_{\mu}^{x x}(x, t)\right\rangle$ is not zero, however up to a small error.

For the other test problems, the situation is the same as the one we describe here. It is possible to find of value for $K_{M}$ by adjusting the largest components of stress and strain tensors (in the limit $t \rightarrow \infty$ ), and there is a small error on some components ( $x x$ and $y y$ in shear load, $x y$ in tension compression load). The values found for $K_{M}$ are given in Tab. 1 . We notice that, up to a $0.4 \%$ error, the value depends neither on the type of boundary conditions, on the number of grains, on the mesh size nor on the orientation distribution sample. Thus the polycrystal obeys an effective constitutive law with $K_{M}=346 \mathrm{MPa}$.

In order to measure the error of the small components of the tensors with respect to the average value, we define some empiric estimators:

- for mixed boundary conditions, $\lim _{t \rightarrow \infty}\left(\left\langle\varepsilon_{\mu}^{x y}\right\rangle /\left\langle\varepsilon_{\mu}^{y y}\right\rangle\right)$;

- $\lim _{t \rightarrow \infty}\left(\left\langle\sigma_{\mu}^{x x}\right\rangle /\left\langle\sigma_{\mu}^{x y}\right\rangle\right)$ for shear load;

- for tension-compression load, $\lim _{t \rightarrow \infty}\left(\left\langle\sigma_{\mu}^{x y}\right\rangle /\left\langle\sigma_{\mu}^{y y}\right\rangle\right)$;

- $\lim _{t \rightarrow \infty}\left(\left\langle\sigma_{\mu}^{x x} \pm \sigma_{\mu}^{x y}\right\rangle / \sigma_{M}^{x y}\right)$ for tension-compression-shear load, boundary conditions being so that $\lim _{t \rightarrow \infty} \sigma_{M}^{x x} \pm \sigma_{M}^{x y}=0$;

The values found for these estimators are given in Tab. 2. One can notice that all errors are small (less than $3 \%$ ), so the effective law is a good approximation of the microscopic model in most of the situations studied. 


\begin{tabular}{|c|c|c|c|c|}
\hline & $\begin{array}{c}30 \text { grains } \\
\text { coarse mesh }\end{array}$ & $\begin{array}{c}30 \text { grains } \\
\text { fine mesh }\end{array}$ & $\begin{array}{c}110 \text { grains } \\
\text { sample 1 }\end{array}$ & $\begin{array}{c}110 \text { grains } \\
\text { sample 2 }\end{array}$ \\
\hline \hline T & 345.6 & & 347.1 & \\
\hline T C & 345.6 & 345.6 & 347.1 & 345.3 \\
\hline S & 347.25 & 347.25 & 344.6 & 344.6 \\
\hline TCS & 346.1 & 346.0 & 346.62 & 345.4 \\
\hline
\end{tabular}

Table 1 - Values of $K_{M}$ for different polycrystals with different loadings (the indicated value is the average on different boundary condition values).

\begin{tabular}{|c|c|c|c|c|}
\hline & $\begin{array}{c}30 \text { grains } \\
\text { coarse mesh }\end{array}$ & $\begin{array}{c}30 \text { grains } \\
\text { fine mesh }\end{array}$ & $\begin{array}{c}110 \text { grains } \\
\text { sample 1 }\end{array}$ & $\begin{array}{c}110 \text { grains } \\
\text { sample 2 }\end{array}$ \\
\hline \hline $\mathrm{T}$ & $2 \%$ & & $0.9 \%$ & \\
\hline $\mathrm{T} \mathrm{C}$ & $2.5 \%$ & $2.5 \%$ & $0.8 \%$ & $1.2 \%$ \\
\hline $\mathrm{S}$ & $2.9 \%$ & $2.9 \%$ & $1.1 \%$ & $1 \%$ \\
\hline $\mathrm{TCS}$ & $0.3 \%$ & $0.3 \%$ & $1.4 \%$ & $1.3 \%$ \\
\hline
\end{tabular}

Table 2 - Values of error estimators for different polycrystals with different loadings.

It is also interesting to compute averages on grains of stress or strain tensors, and not on the whole polycrystal. We want to know whether these averages are similar from one grain to another one, or very different. Let us focus on the tension-compression-shear load. At each time step, we compute, for each grain, the average over the grain of $\left(\varepsilon_{\mu}^{v p}\right)^{y y}$ and of $\tilde{\sigma}_{\mu}^{y y}(\tilde{\sigma}$ is the deviatoric part of $\sigma)$. We work with the viscoplastic strain tensor and the deviatoric stress tensor since these are the natural variables for the viscoplastic term of the constitutive law. Results are given in Figs. 3 and 4. At the beginning, the averages for all grains are the same: even without any yield stress, the viscous flow can be neglected, and due to the uniform elasticity assumption, all grains give the same result. As the viscoplastic term increases, grain responses become heterogeneous. According to classical results in elasto-plasticity [9], the first set of points on Fig. 4, corresponding to a very low macroscopic viscoplastic strain (around 0.0002), are aligned along a line, the slope of which is not far from the shear modulus. 


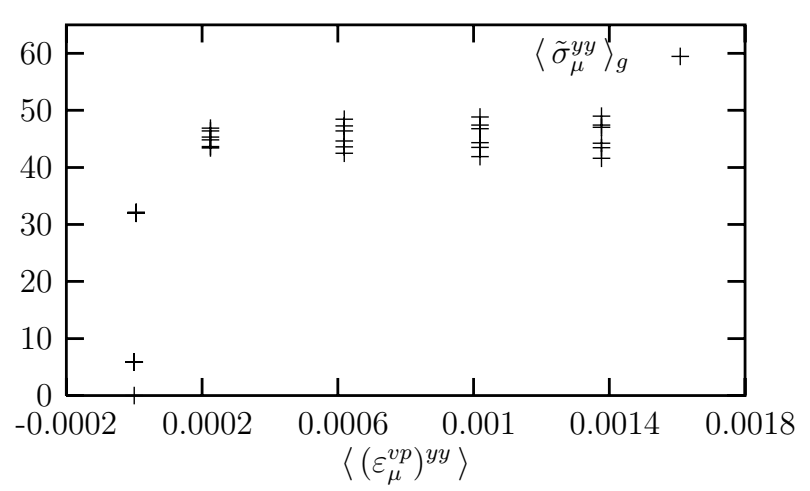

Figure 3 - Evolution of $\left\langle\tilde{\sigma}_{\mu}^{y y}\right\rangle_{g}$ as a function of $\left\langle\left(\varepsilon_{\mu}^{v p}\right)^{y y}\right\rangle$ for the 30 grain polycrystal, tension-compression-shear load $\left(\langle\cdot\rangle_{g}\right.$ is the average over the grain).

Like for self-consistent approaches, the slope decreases during the loading (see the three sets of points for a macroscopic viscoplastic strain of $0.0006,0.0010$ and 0.0014 ), but one can also observe an additional heterogeneity. This kind of curves can be used to calibrate phenomenological models with uniform stress and strain in each phase.

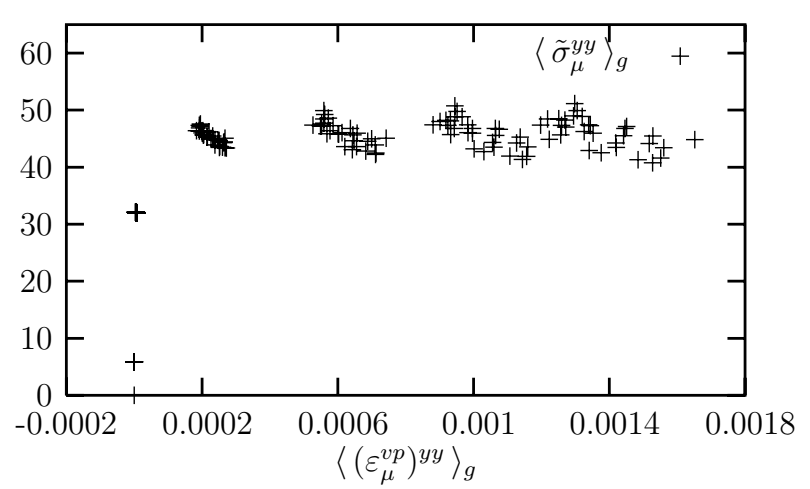

Figure 4 - Evolution of $\left\langle\tilde{\sigma}_{\mu}^{y y}\right\rangle_{g}$ as a function of $\left\langle\left(\varepsilon_{\mu}^{v p}\right)^{y y}\right\rangle_{g}$ for the 30 grain polycrystal, tension-compression-shear load $\left(\langle\cdot\rangle_{g}\right.$ is the average over the grain).

So far, we have just compared responses in the limit $t \rightarrow \infty$ (in this limit, the elastic part of the constitutive law cancells). We may also compare responses during the whole load process, to check whether microscopic and effective laws agree only in the viscoplastic limit or also when elastic and viscoplastic terms are 
of the same order of magnitude. We make such a comparison in Fig. 5. For the other test problems, the situation is alike: the effective law is in good agreement with the microscopic law (the difference is smaller than $1 \%$ ).
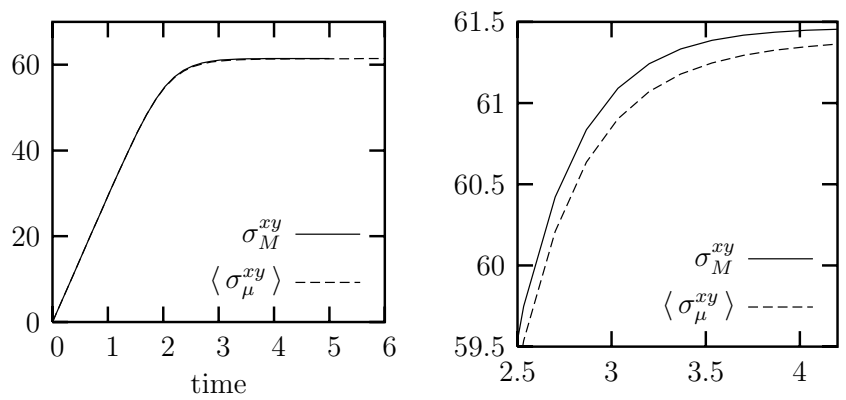

Figure 5 - Transient response of the 30 grain polycrystal, shear load. The effective law is in good agreement with the microscopic law (left). On the right-hand side, a zoom on the region where there are some differences.

This numerical result is very surprising. Starting from a microscopic constitutive law which is time-dependent and involves two potentials, we split it into two terms. We apply separately on each of them a procedure which is based on stationary calculus of variations. We fit $K_{M}$ on the long-time limit of the system, which corresponds to the viscoplastic regime. The numerical result is that the effective law is in agreement with the microscopic one both in stationary and transient regime! We acknowledge the fact that there is no rigorous reason for this success: we just observe that the two laws are consistent.

\section{Conclusions}

We have dealt in this article with a simple model of a 2D heterogeneous elastoviscoplastic polycrystal, for which no theoretical results on the effective law are available. We have succeeded in numerically identifying an effective law. We observe that this effective law is consistent with the microscopic law in both the stationary and transient regime, although it has been obtained by a homogenization procedure designed for stationary problems. We are unfortunately unable to provide any explanation for this fact but are currently working in that direction. 


\section{Acknowledgments}

I would like to thank Claude Le Bris for many insights incorporated in this article and for his very careful reading of the manuscript, as well as Georges Cailletaud and Renaud Masson for having introduced me to the field and for their very helpful suggestions.

\section{REFERENCES}

[1] F. Barbe, L. Decker, D. Jeulin and G. Cailletaud, Intergranular and intragranular behaviour of polycrystalline aggregates, Part 1: F.E. model, Int. J. Plasticity, 17 (2001), 513-536.

[2] F. Barbe, S. Forest and G. Cailletaud, Intergranular and intragranular behaviour of polycrystalline aggregates, Part 2: Results, Int. J. Plasticity, 17 (2001), 537-563.

[3] J. Besson, G. Cailletaud and J.-L. Chaboche, S. Forest, Mécanique non linéaire des matériaux, Hermès, Etudes en mécanique des matériaux et des structures, Paris, (2001).

[4] M. Bornert and P. Ponte Castañeda, Second-order estimates of the self-consistent type for viscoplastic polycrystals, Proc. R. Soc. Lond. A 454 (1998), 3035-3045.

[5] J.-L. Chaboche and G. Cailletaud, Integration methods for complex plastic constitutive equations, Comput. Methods Appl. Mech. Engrg. 133 (1996), 125-155.

[6] P.G. Ciarlet, Mathematical Elasticity, North-Holland, Amsterdam, (1988).

[7] J.D. Eshelby, The determination of the elastic field of an ellipsoidal inclusion and related problems, Proc. R. Soc. Lond. A 421 (1957), 376-396.

[8] R.V. Kohn and T.D. Little, Some model problems of polycrystal plasticity with deficient basic crystals, SIAM J. Appl. Math, 59 (1) (1998), 172-197.

[9] E. Kröner, Zur plastischen Verformung des Vielkristalls (On the plastic deformation of polycrystals), Acta. Met. 9 (1961), 155-161.

[10] P. Le Tallec, Numerical Methods for Nonlinear Three-dimensional Elasticity, Handbook of Numerical Analysis vol. III, 465-624, Eds. P.G. Ciarlet, J.L. Lions, North-Holland, Amsterdam, (1994).

[11] P. Ponte Castañeda, Exact second-order estimates for the effective mechanical properties of nonlinear composite materials, J. Mech. Phys. Solids, 44 (6) (1996), 827-862.

[12] P. Ponte Castañeda and P. Suquet, Nonlinear composites, Advances in Applied Mechanics, 34 (1998) 171-302.

[13] P. Ponte Castañeda and J.R. Willis, Variational second-order estimates for nonlinear composites, Proc. R. Soc. Lond. A 455 (1999), 1799-1811. 